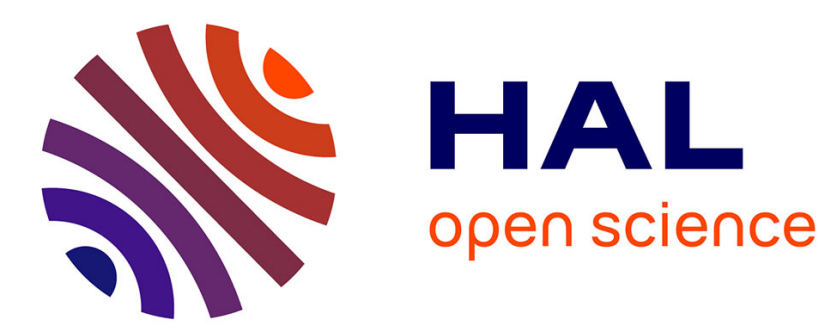

\title{
Non-trivial exponents in the zero temperature dynamics of the 1D Ising and Potts models
}

\author{
B. Derrida, A.J. Bray, C. Godrèche
}

\section{To cite this version:}

B. Derrida, A.J. Bray, C. Godrèche. Non-trivial exponents in the zero temperature dynamics of the 1D Ising and Potts models. Journal of Physics A: Mathematical and Theoretical, 1994, 27, pp.L357-L361. 10.1088/0305-4470/27/11/002 . hal-00165918

\section{HAL Id: hal-00165918 https://hal.science/hal-00165918}

Submitted on $30 \mathrm{Jul} 2007$

HAL is a multi-disciplinary open access archive for the deposit and dissemination of scientific research documents, whether they are published or not. The documents may come from teaching and research institutions in France or abroad, or from public or private research centers.
L'archive ouverte pluridisciplinaire HAL, est destinée au dépôt et à la diffusion de documents scientifiques de niveau recherche, publiés ou non, émanant des établissements d'enseignement et de recherche français ou étrangers, des laboratoires publics ou privés. 
February 14, 2001

\title{
NONTRIVIAL EXPONENTS IN THE ZERO TEMPERATURE DYNAMICS OF THE 1- $d$ ISING AND POTTS MODELS
}

\author{
B. Derrida ${ }^{1,2}$, A.J. Bray ${ }^{3}$ and C. Godrèche ${ }^{4}$ \\ Isaac Newton Institute for Mathematical Sciences, \\ 20 Clarkson Road, Cambridge CB3 0EH, U.K.
}

\begin{abstract}
We consider the Glauber dynamics of the $q$-state Potts model in one dimension at zero temperature. Starting with a random initial configuration, we measure the density $r_{t}$ of spins which have never flipped from the beginning of the simulation until time $t$. We find that for large $t$, the density $r_{t}$ has a power law decay $\left(r_{t} \sim t^{-\theta}\right)$ where the exponent $\theta$ varies with $q$. Our simulations lead to $\theta \simeq .37$ for $q=2$, $\theta \simeq .53$ for $q=3$ and $\theta \rightarrow 1$ as $q \rightarrow \infty$.
\end{abstract}

PACS 02.50, 05.20

Short Title: Zero Temperature Dynamics of the Potts model

to be submitted to Journal of Physics A Letters

Permanent addresses:

${ }^{1}$ Laboratoire de Physique Statistique, Ecole Normale Supérieure, 24 rue Lhomond, 75231 Paris cedex 05, France.

${ }^{2}$ Service de Physique Théorique, CE Saclay, F91191 Gif sur Yvette, France.

${ }^{3}$ Department of Theoretical Physics, The University, Manchester M13 9PL, UK.

${ }^{4}$ Service de Physique de l'Etat Condensé, CE Saclay, F91191 Gif sur Yvette, France. 
The study of irreversible processes has led to the discovery of a large variety of non-trivial critical behaviors. Such processes include reaction-diffusion problems [1], [2], [3], [4], [5], [6], random sequential parking or fragmentation models [7], [8], [9], [10], [11], domain growth models [12] and the kinetic roughening of interfaces [13], [14].

In an earlier work [12], we have studied a simple deterministic model of domain growth in one dimension. The system consists of a sequence of intervals along the line. At each time step, the two closest domain walls in the system merge and annihilate whereas the other domain walls do not move. Starting with random initial lengths for the domains, we showed that the fraction $r_{t}$ of the line which has not been crossed by any domain wall up to time $t$ decays with a power law $r_{t} \sim l(t)^{-\theta^{\prime}}$ where $l(t)$ is the average length of the domains at time $t$ and $\theta^{\prime} \simeq .1750759 \ldots$ is a non-trivial exponent, the solution of a transcendental equation (notice that in reference [12], we used the exponent $\beta$ related to our present $\theta^{\prime}$ by $\beta=1-\theta^{\prime}$ ). As for other critical exponents, $\theta^{\prime}$ is universal in the sense that it is the same for all initial conditions (provided that the correlations in the initial condition are short range).

In the present work, we investigate the same kind of question for similar models with stochastic dynamics: the one dimensional Ising model at zero temperature and its generalisation to the general $q$-state Potts model. In contrast to our previous results [12], our approach here will be mostly numerical because so far we have not been able to develop an analytical approach.

Our simulation of the dynamics of the one-dimensional Ising model with ferromagnetic interactions at zero temperature is done for a system of $N=100000$ spins with periodic boundary conditions. We start with a random initial spin configuration (each spin is initially + or - with equal probability). The updating rule to go from configuration $C_{t}$ at time $t$ to the next configuration $C_{t+\Delta t}$ at time $t+\Delta t$ is done by choosing one spin $i$ at random. This spin is aligned with its two neighbors 
if these two neighbors are parallel or is flipped at random if the two neighbors are opposite [15]. We take here our time step $\Delta t=1 / N$ so that each spin in the system is updated on average once per unit of time. Then we count the total number $R_{t}$ of spins which never flipped until time $t$ (the number of non-moving spins was shown to have interesting properties in some deterministic voter models [16]). The density $r_{t}$ of these spins is thus $r_{t}=R_{t} / N$.

Our results done from time $t=0$ until time $t=10000$ are shown on figure 1 and indicate that $r_{t}$ has a power law decay

$$
r_{t} \sim t^{-\theta}
$$

with

$$
\theta_{\text {Ising }} \simeq .37
$$

The log-log plot gives a straight line from time $t \simeq 3$ to $t \simeq 10000$ and we estimate the error bar to be about .02 . For the 1-d Ising model, it is well known [17], [18] that the average length $l(t)$ of domains increases as $t^{1 / 2}$. Therefore, one could also write $r_{t} \sim l(t)^{-\theta^{\prime}}$ with $\theta^{\prime}=2 \theta$.

We repeated the same calculation for the $q$-state Potts model [19], [20] again for a system of $N=100000$ spins and for times up to 10000 . In this case each spin is given initially a random integer value between 1 and $q$. Then the updating rule is that at each time step $\Delta t$, a site $i$ is chosen at random and the spin $S_{i}$ becomes equal to the value of either its left neighbor or its right neighbor with equal probability. As in the Ising case, we take periodic boundary conditions.

Our results $\left(r_{t}\right.$ versus $\left.t\right)$ obtained for $q=3,5,10$ and $\infty$ are shown on Figure 1 ( for $q=\infty$, all the spins in the initial configuration are in a different state; one can take for example $S_{i}=i$ ). For the Potts model, as for the Ising case, they indicate that $r_{t}$ decays like a power law and our estimates for the exponent $\theta$ are:

$$
\begin{aligned}
& \theta_{\mathrm{q}=3} \simeq .53 \\
& \theta_{\mathrm{q}=5} \simeq .70
\end{aligned}
$$




$$
\begin{aligned}
& \theta_{\mathrm{q}=10} \simeq .82 \\
& \theta_{\mathrm{q}=\infty} \simeq 1.0
\end{aligned}
$$

Here again the log-log plot gives a straight line for times $t>5$. Since $r_{t}$ decreases as $q$ increases, the number of spins which have never flipped becomes so small, for the system size that we considered, that the data are noisy. As for the Ising case we estimate our error bar to be about \pm .02 .

We see that the exponent $\theta$ seems to vary with $q$. This is reminiscent of what we already found in the simple geometrical growth model of reference [12] where $\theta^{\prime}$ was varying from $.1750759 \ldots$ to 1 when $q$ changes from 2 to $\infty$.

We did not find a way of calculating the exponent $\theta$ for general values of $q$. The case $q=\infty$ can however be easily understood from a simple domain wall argument. When $q=\infty$, one can think of the domain walls as being particles on the line performing a Brownian motion with the reaction rule that when two particles meet, they merge into one particle: $A+A \rightarrow A$. Any given site does not flip between time 0 and time $t$ if and only if it has been visited by neither the first particle on its right nor the first particle on its left. It is a well known fact of random walk theory that the probability $p_{t}$ that a site is not visited by a random walker at its right decays like $p_{t} \sim t^{-1 / 2}$. Then if we want a site to be visited neither by the particle at its right nor by the particle at its left, one gets $r_{t}=p_{t}^{2}$ and this leads to $\theta=1$. Unfortunately, it is not easy to extend this idea to general $q$ because depending on the Potts states of domains, one has either $A+A \rightarrow 0$ or $A+A \rightarrow A$ and this means that sometimes the closest particle at the right (or at the left) of a given site might disappear before it reaches that site.

One can measure the number of spins which flipped only once between time 0 and time $t$. Figure 2 shows for the 1-d Ising model a log-log plot of the density of spins which did not flip and of the density of spins which flipped only once. The results indicate that the power law decay is the same for the two quantities and it is reasonable to expect that the density of spins which flip exactly $n$ times with $n$ 
fixed as $t \rightarrow \infty$ would decay with the same exponent.

One can also try to repeat the above calculations in higher dimensions. We present in Figure 3 the results obtained for the 2-d Ising model. In dimension 2, we used a system of $300^{2}$ sites and the range of time was 0 to 10000 . We observe again a power law decay of the density $r_{t}$ of spins which have never flipped, with an exponent $\theta \simeq .22 \pm .03$. This estimate is done over the range $5 \leq t \leq 1000$. As usual the short time results should be eliminated because they are non-universal. Also, we believe that the late time data are not too reliable because the characteristic length of the domains starts to be comparable to the system size. One could try to measure the exponent $\theta$ in higher dimension but we expect finite-size effects to become more and more serious as the dimension increases. This is again because the characteristic length of domains increases as $t^{1 / 2}$ in all dimensions, whereas the linear dimension of the system one can simulate obviously decreases with dimension. So the range of times which are not affected by finite size effects decreases with dimension.

In this letter, we have presented numerical results indicating that for the 1-d Ising and Potts models, the density of spins which have never flipped has an interesting power law decay with time and the exponents seem to be non-trivial. In the case $q=\infty$, a simple argument proves that the exponent $\theta$ is equal to 1 , in agreement with the numerical data.

When one thinks of the dynamics as a Brownian motion of the domain walls, one realizes that the problem is closely related to reaction diffusion models [18], [20]. For example the Ising case corresponds to an initial situation where all sites are occupied by $B$ particles and where the domain walls are represented by $A$ particles. The dynamics is that the particles $A$ are random walkers whereas particles $B$ do not move, with the reactions $A+A \rightarrow 0$ (the annihilation of domain walls) and $A+B \rightarrow A+0$ as the non-moving sites disappear whenever they are visited by domain walls. The case of the Potts model corresponds to having in addition the possibility that $A+A \rightarrow A$. A great deal of work has been done recently to 
understand the critical exponents which characterize the long time behavior of the density in these reaction diffusion models [1], [2], [4], [3], [5], [6], [21] and it would be interesting to know whether the methods [22], [23], [24], [25] which have been developped for these systems could be used to predict the values of $\theta$ that we have measured from our data shown in figure 1. The higher dimensional cases however would require a different approach as the domain walls are extended objects (lines or surfaces) rather than points.

As the case $q=\infty$ is understood, one could try to calculate the exponent $\theta$ using a large $q$ expansion. Another interesting question would be to extend the notion of non-moving spins to non-zero temperature. At non-zero temperature, the number of non-moving spins decays exponentially in all dimensions. However when the characteristic size of domains becomes larger than the equilibrium correlation length, one can associate to each site in the system the phase it is in (except perhaps for the sites too close to a domain boundary) and one can count the number of time steps a site spends in a given phase.

Acknowledgements: We thank the Isaac Newton Institute, Cambridge, UK where this work was carried out. We had useful discussions with J.L. Cardy, M. Droz, V. Hakim, J. Kertész, E.R. Speer and J. E. Steif.

\section{References}

[1] Z. Rácz, Phys. Rev. Lett. 55, 1707 (1985)

[2] R. Schoonover, D. Ben-Avraham, S. Havlin, R. Kopelman and G.H. Weiss, Physica A 171, 232 (1991)

[3] F. Leyvraz and S. Redner, Phys. Rev. Lett. 66, 2168 (1991)

[4] F. Leyvraz and S. Redner, Phys. Rev. A46, 3132 (1992) 
[5] S. Cornell, M. Droz and B. Chopard, Physica A 188, 322 (1992)

[6] S. Cornell, M. Droz, Phys. Rev. Lett. 70, 3824 (1993)

[7] Y. Pomeau, J. Phys. A13, L193 (1980)

[8] R. M. Ziff and E. D. McGrady, Macromolecules 19, 2513 (1986)

[9] J.P. Sherwood, J. Phys. A23, 2827 (1990)

[10] G. Tarjus and P. Viot, Phys. Rev. Lett. 67, 1875 (1991)

[11] P. L. Krapivsky, J. Stat. Phys. 69, 135 (1992)

[12] A.J. Bray, B. Derrida and C. Godrèche, preprint 94: 'Nontrivial algebraic decay in a soluble model of coarsening'

[13] S.F. Edwards, D. Wilkinson, Proc. Roy. Soc. A381, 17 (1982)

[14] M. Kardar, G. Parisi, Y. Zhang, Phys. Rev. Lett. 56, 889 (1986)

[15] R.J. Glauber, Journ. Math. Phys. 4, 294 (1963)

[16] R. Durrett and J.E. Steif, Annals of Probability 21, 232 (1993)

[17] A.J. Bray, J. Phys. A23, L67 (1990)

[18] F. Family and J.G. Amar, J. Stat. Phys. 65, 1235 (1991)

[19] M. Droz, J. Kamphorst Leal da Silva, A. Malaspinas and J. Yeomans, J. Phys. A19, $2671(1986)$

[20] B. Derrida, C. Godrèche, I. Yekutieli, Phys. Rev. A 44, 6241 (1991)

[21] D. Toussaint, F. Wilczek, J. Chem. Phys. 78, 2642 (1983)

[22] M. Bramson and J.L. Lebowitz, J. Stat. Phys. 62, 297 (1991)

[23] M. Doi, J. Phys. A9, 1479 (1976) 
[24] L. Peliti, J. Physique 46, 1469 (1985)

[25] B. P. Lee, Oxford preprint 93: 'Renormalization group calculation for the reaction $k A \rightarrow 0$ 


\section{Figure Captions}

1. Figure 1: $\log -\log$ plot of the density $r_{t}$ of spins which have never flipped versus time for several one dimensional Potts models (of course, $q=2$ corresponds to the Ising model). For all choices of $q$, the number of states of the Potts model, $r_{t}$ has a power-law decay with an exponent which depends on $q$. For large $q$, the large $t$ data become noisy because the system size is not big enough.

2. Figure 2: $\log$-log plot of the density of spins which have never flipped and of the density of spins which flipped only once, for the 1-d Ising model. These two densities seem to have the same power law decay in the long time limit.

3. Figure 3: the density $r_{t}$ of spins which have never flipped for the 2-d Ising model. 